\title{
QUALIDADE DAS SEMENTES DE SOJA COM A APLICAÇÃO DE DIFERENTES DOSES DE MOLIBDÊNIO E COBALTO ${ }^{1}$
}

\author{
ANDRÉ LUIS GOLO², CLAUDINEI KAPPES², MARCO ANTONIO CAMILLO DE CARVALHO; \\ OSCAR MITSUO YAMASHITA ${ }^{4}$
}

\begin{abstract}
RESUMO - O presente trabalho foi desenvolvido objetivando avaliar o efeito da aplicação de diferentes doses de cobalto (Co) e molibdênio (Mo) via foliar e a inoculação das sementes com Bradyrhizobium japonicum, sobre as características produtivas da cultura da soja e na qualidade de suas sementes. O estudo foi realizado no ano agrícola de 2005/06, no município de Santa Carmem, MT, em Latossolo Vermelho Amarelo distrófico. O delineamento experimental foi de blocos casualizados em esquema fatorial $6 \mathrm{X}$ 2, perfazendo 12 tratamentos com quatro repetições cada. Os tratamentos foram constituídos pela combinação de seis doses do produto comercial Quimifol CoMo Plus (0,0; 0,25; 0,5; 1,0; 1,5; e 2,0 L ha-1), aplicados via foliar, com e sem a inoculação das sementes com inoculante comercial de Bradyrhizobium japonicum (Rizo-Liq), formulação líquida. Com base nos resultados, concluiu-se que: a inoculação das sementes aumenta o número de vagens por planta e o número de sementes por planta; as doses de Co e Mo afetam a massa de 100 sementes e a produtividade; a inoculação não apresenta efeito significativo sobre a produtividade; a aplicação de Co e Mo na dose de 2 L ha-1 em sementes inoculadas promove melhoria na qualidade fisiológica das sementes; na ausência de inoculação, o aumento da doses de Co e Mo ocasiona diminuição na qualidade fisiológica das sementes.
\end{abstract}

Termos para indexação: inoculante; micronutrientes; vigor; Bradyrhizobium japonicum.

\section{QUALITY OF SOYBEAN SEEDS PRODUCED WITH DIFFERENT DOSES OF MOLYBDENUM AND COBALT}

\begin{abstract}
The present work study assessed the effect of foliar application of different doses of cobalt (Co) and molybdenum (Mo), and seed inoculation with Bradyrhizobium japonicum on the productive characteristics of the soybean crop and the quality of its seeds. The study was carried out in 2005/06, in a Red-Yellow Latosol (Hapludox) dystrophic soil, in the city of Santa Carmen, MT. The experimental design was a randomized block in a 6 X 2 factorial design, with a combination of 12 treatments with four replications for each treatment. The treatments resulted from the combination of six doses of the product Quimifol CoMo Plus $(0.0 ; 0.25 ; 0.5 ; 1.0 ; 1.5$; and $2.0 \mathrm{~L}$ ha- 1$)$, applied foliarly, with and without inoculation of the seeds with a liquid formulation of Bradyrhizobium japonicum, RizoLiq. Based on the results, the following conclusions were drawn: seed inoculation with Bradyrhizobium increased the number of pods per plant and the number of seeds per plant;
\end{abstract}

${ }^{1}$ Submetido em 04/07/2007, Aceito para publicação em 18/04/2008. Trabalho de Conclusão de Curso do primeiro autor apresentada a UNEMAT.

${ }^{2}$ Graduandos do Departamento de Agronomia, Universidade do Estado de Mato Grosso (UNEMAT), Caixa postal 324, 78580-000, Alta Floresta, andre_golo@hotmail.com, code.agro@hotmail.com;
${ }^{3}$ Eng. Agr ${ }^{\circ}$. Dr. Coordenador do Campus Universitário de Alta Floresta, UNEMAT, Caixa posta 324, 78580-000, Alta Floresta, marcocarvalho@ unemat.br;

${ }^{4}$ Eng. Agr ${ }^{0}$. MSc. Professor Assistente, Departamento de Agronomia, UNEMAT, Caixa posta 324, 78580-000, Alta Floresta, yama@unemat.br. 
the doses of CoMo affected the mass of 100 seeds and the grain yield; inoculation did not result in increases in grain yield; the application of $2 \mathrm{~L}$ ha-1 CoMo to inoculated seed improved the seed physiological quality; in the absence of inoculation, increasing doses of CoMo caused decrease in seed physiological quality.

Index terms: inoculant; micronutrients; seed vigor; Bradyrhizobium japonicum.

\section{INTRODUÇÃO}

A soja [Glycine max (L.) Merrill] é a cultura anual de maior expressão econômica no Brasil, sendo que seu cultivo se tornou viável, em grande parte, devido à sua capacidade de fixar o nitrogênio atmosférico para a sua nutrição, em associação com a bactéria Bradyrhizobium japonicum (Vargas et al., 1994). A inoculação das sementes de soja é o primeiro passo para a obtenção de boa nodulação e, conseqüentemente, ao adequado suprimento de nitrogênio às plantas de soja através da fixação biológica de nitrogênio (FBN).

A fixação biológica é seriamente afetada quando ocorre deficiência de molibdênio (Mo), tendo em vista que este nutriente faz parte da enzima nitrogenase, responsável pelo processo da fixação (Marschner, 1986). O mesmo ocorre com a deficiência do cobalto $(\mathrm{Co})$, que influencia a absorção de nitrogênio, porque participa da estrutura da vitamina B12, necessária à síntese de leghemoglobina, que determina a atividade dos nódulos (Mengel e Kirkby, 2001).

Uma das etapas mais importantes na produção de soja é a obtenção de sementes de alta qualidade fisiológica para que possam ser utilizadas pelos agricultores no estabelecimento de suas lavouras (Rocha et al., 1984). Em campos de produção de sementes de soja, experimentos relacionados à adubação e à nutrição das plantas são escassos, de modo que o emprego de fertilizantes é feito com base nos resultados obtidos para a produção de grãos (Carvalho e Nakagawa, 2000).

As plantas adubadas de modo adequado e equilibrado apresentam condições de produzir maior quantidade de sementes, aliadas à melhor qualidade, podendo resistir mais facilmente às adversidades que podem surgir no período de produção (Sá, 1994). No Brasil, são poucos os estudos sobre os efeitos dos micronutrientes na produtividade e sobre a qualidade fisiológica de sementes de soja. Além disso, tais estudos têm se concentrado mais em Phaseolus vulgaris e, especialmente, com Mo (Sfredo et al., 1997).

O presente trabalho foi desenvolvido objetivando avaliar o efeito da aplicação de diferentes doses de cobalto (Co) e molibdênio (Mo) via foliar e a inoculação das sementes com Bradyrhizobium japonicum, sobre as características produtivas da cultura da soja e a qualidade fisiológica das suas sementes.

\section{MATERIAIS E MÉTODOS}

O experimento foi conduzido em campo comercial da Fazenda Golo, no município de Santa Carmem, MT, situada nas coordenadas geográficas de $12^{\circ} 01^{\prime} 40^{\prime}$ S e $55^{\circ} 14^{\prime}$ $35^{\prime} \mathrm{O}$, com altitude de $365 \mathrm{~m}$. O clima da região, segundo classificação de Koppen, é do tipo Aw, ou seja, tropical chuvoso, com um período seco definido. O solo é classificado como Latossolo Vermelho Amarelo distrófico, de textura Argilosa, do qual foram coletadas amostras na camada de 0 a 0,2 m, cujas características químicas estão apresentadas na Tabela 1.

$\mathrm{O}$ delineamento experimental utilizado foi o de blocos casualizados em esquema fatorial 6 X 2, perfazendo 12 tratamentos dotados de quatro repetições cada. Os tratamentos foram constituídos pela combinação de seis doses $(0,0 ; 0,25$; 0,5; 1,0; 1,5; e 2,0 $\mathrm{L} \mathrm{ha}^{-1}$ ) do produto comercial Quimifol CoMo Plus, contendo $1 \%$ de cobalto e $6 \%$ de molibdênio aplicados via foliar, com e sem a aplicação do inoculante comercial de Bradyrhizobium japonicum (Rizo-Liq), formulação líquida. O inoculante possuía as estirpes SEMIA 587, SEMIA 5019 e SEMIA 5028, tendo $1,5 \times 10^{9}$ células viáveis $\mathrm{mL}^{-1}$, sendo aplicado na dose de $140 \mathrm{~mL}$ por $50 \mathrm{~kg}$ de sementes. As fontes de Co e Mo contidas no produto utilizado são o sulfato de cobalto e o molibdato de sódio, respectivamente, sendo o Co quelatizado e, assim, permitindo a aplicação do produto via foliar. O CoMo foi aplicado via foliar, quando a cultura se encontrava no estádio V4, aos 21 dias após a emergência (DAE), com pulverizador costal de $20 \mathrm{~L}$, com ponta cônica na vazão de 220 litros de calda ha ${ }^{-1}$.

As parcelas foram constituídas por nove linhas de 6,0 
m de comprimento espaçadas a 0,45 m entre si. A área útil de cada parcela foi constituída pelas 3 linhas centrais, desprezando-se $1,0 \mathrm{~m}$ em ambas as extremidades de cada linha, perfazendo área útil de 5,4 $\mathrm{m}^{2}$.

TABELA 1. Resultados das análises químicas para macronutrientes, micronutrientes e resultados complementares do solo da área do experimento localizado na Faz. Golo, município de Santa Carmem, MT (2005). ${ }^{1}$

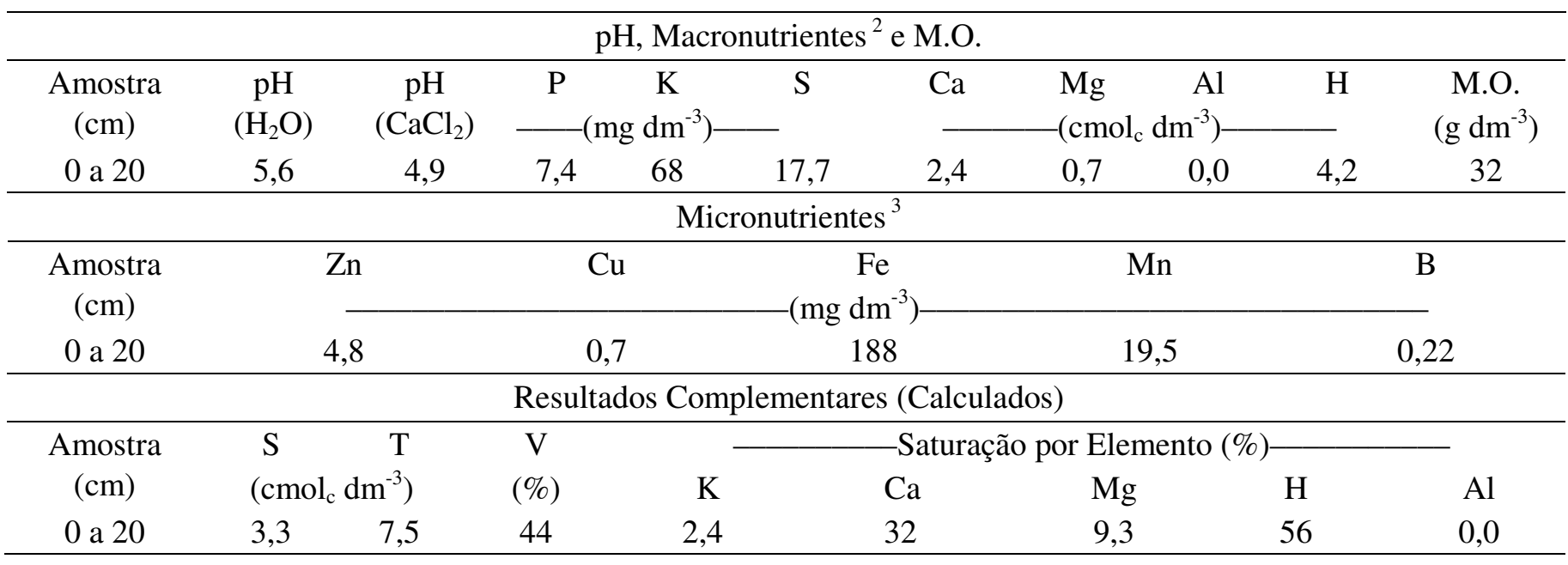

${ }^{1}$ Análises realizadas no laboratório Plante Certo, Várzea Grande, MT.

${ }^{2}$ Extratores: P e K: Mehlich I; Ca, Mg e Al: KCI 1 N; H + Al: Acetato de cálcio pH = 7,0.

${ }^{3}$ Extratores: $\mathrm{Zn}, \mathrm{Cu}, \mathrm{Fe}$ e Mn: $\mathrm{H}_{2} \mathrm{SO}_{4}$ 0,025N + $\mathrm{HCl}$ 0,05N; S: Fosfato de cálcio; B: HCl 0,05N (Água quente).

O manejo do solo foi realizado por cultivo mínimo. A semeadura ocorreu no dia 15 de outubro de 2005. As sementes foram semeadas à profundidade de $3 \mathrm{~cm}$, com população em

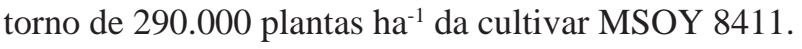

Utilizou-se em adubação de base $500 \mathrm{~kg} \mathrm{ha}^{-1}$ da fórmula 00-18-18 (0 kg de N, $90 \mathrm{~kg}$ de $\mathrm{P}_{2} \mathrm{O}_{5}$ e $90 \mathrm{~kg}$ de $\mathrm{K}_{2} \mathrm{O}$ ) nos sulcos de plantio, ao lado e abaixo da linha de semeadura, sendo a adubação de semeadura calculada de acordo com as características químicas do solo, levando-se em consideração as recomendações de Novais (1999).

Os tratos culturais constituíram-se de aplicações dos herbicidas Imazethapyr na dose de $1 \mathrm{~L} \mathrm{ha}^{-1}$ (p.c.) e FluasifopP-butil na dose de 0,6 L ha"-1 (p.c.), para o controle de plantas daninhas. Em relação ao controle de insetos ocorreram as aplicações dos inseticidas Lufenuron + Profenofós na dose de $0,125 \mathrm{~L} \mathrm{ha}^{-1}$ (р.c.), visando o controle de lagartas, e duas aplicações do inseticida Metamidofós na dose de 0,8 L ha ${ }^{-1}$ (p.c.), para o controle de percevejos, de acordo com amostragens periódicas realizadas na lavoura.

Com o objetivo de uniformizar a maturação das plantas, aos 105 dias após a semeadura (DAS), foi realizada a dessecação da cultura com a utilização do dessecante Diquat, na dose de $1 \mathrm{~L} \mathrm{ha}^{-1}$ (р.c.). A colheita manual foi realizada uma semana após a dessecação da cultura, no dia 01 de fevereiro de 2006. No dia seguinte à colheita, foi realizada a debulha manual das plantas e as sementes, depois de limpas, foram acondicionadas em sacos de papel e identificadas.

Por ocasião da maturação das plantas, foram coletadas 10 plantas dentro de uma das linhas da área útil, para realização das avaliações onde foram determinadas: altura média de plantas: medida entre o colo da planta e o ápice do caule, com o auxilio de uma trena milimetrada; altura de inserção da primeira vagem: medida da distância entre o colo da planta e a inserção da primeira vagem, com o auxílio de uma trena milimetrada; número médio de vagens e de grãos por planta: determinado pela relação entre o número de vagens e de grãos rigorosamente contados, dividido pelo número de plantas avaliadas; número de grãos por vagem: determinado dividindo-se o número médio de grãos pelo número médio de vagens por planta.

No período de maturação, também foram determinadas as produtividades de grãos e a massa de 100 sementes, ambas a $13 \%$ de umidade.

A qualidade fisiológica das sementes foi avaliada pelos testes de: germinação, realizado com quatro rolos (subamostras) de 50 sementes cada, selecionadas ao acaso 
da produção, por repetição por tratamento, à temperatura de $25^{\circ} \mathrm{C}$, utilizando-se de papel Germitest, como substrato, o qual foi umedecido com quantidade de água destilada equivalente a 2,5 vezes o seu peso e as contagens realizadas aos cinco e aos oito dias após a montagem do mesmo, conforme as Regras para Análise de Sementes (Brasil, 1992); Índice de velocidade de germinação (IVG), realizado conjuntamente ao teste de germinação, sendo realizadas as contagens do número de plântulas normais aos cinco e aos oito dias após a montagem do mesmo. Posteriormente foi calculada a velocidade de germinação aplicando-se a fórmula proposta por Vieira e Carvalho (1994): " $\mathrm{Vg}=\mathrm{N}_{1} / \mathrm{D}_{1}+\mathrm{N}_{2}$ / $\mathrm{D}_{2}+\ldots+\mathrm{N}_{\mathrm{n}} / \mathrm{D}_{\mathrm{n}}$, onde: $\mathrm{Vg}=$ Velocidade de Germinação; $\mathrm{N}_{1}, \mathrm{~N}_{2}$ e $\mathrm{N}_{\mathrm{n}}=$ número de plântulas germinadas aos $1,2 \mathrm{e} \mathrm{n}$ dias após a implantação do teste; $\mathrm{D}_{1}, \mathrm{D}_{2}$ e $\mathrm{D}_{\mathrm{n}}=$ número de dias após montagem do teste; Primeira contagem, realizada em conjunto com o teste de germinação, computando-se o número de plântulas normais obtidas aos cinco dias após a instalação, conforme Marcos-Filho et al. (1987); Teste de frio, onde foram utilizadas quatro repetições de 50 sementes selecionadas ao acaso da produção por tratamento, que foram distribuídas sobre o papel Germitest previamente umedecido da mesma maneira que para o teste de germinação. Após a montagem, os rolos foram colocados em sacos plásticos e vedados, sendo então mantidos em câmara fria a $10^{\circ} \mathrm{C}$ por sete dias, de acordo com Carvalho et al. (2000). Em seguida, os rolos foram transferidos a um germinador ajustado a $25^{\circ} \mathrm{C}$, durante quatro dias, procedendo-se, em seguida, a avaliação do número de plântulas normais, conforme Barros (1999).

Os resultados obtidos foram submetidos à análise de variância, utilizando-se o programa SANEST (Zonta e Machado, 1987). Os fatores qualitativos foram comparados pelo teste de Tukey a $5 \%$ de probabilidade, de acordo com Gomes (1984), e os fatores quantitativos foram submetidos à análise de regressão polinomial.

\section{RESULTADOS E DISCUSSÃO}

Com os resultados da análise estatística para os parâmetros altura de planta, altura de inserção de $1^{\text {a }}$ vagem, número de vagens por planta e número de sementes por planta sob diferentes doses de Co e Mo, com e sem a inoculação com B. japonicum, estão apresentados na Tabela 2. Foi possível verificar que essas características não foram influenciadas pela aplicação das diferentes doses de CoMo $(\mathrm{P} \leq 0,05)$.

Esses resultados estão de acordo com os obtidos por Meschede etal. (2004) que também não observaram diferenças significativas nos resultados obtidos com a variável altura de plantas e altura de inserção de inserção de $1^{\mathrm{a}}$ vagem, quando foi realizada a aplicação de Co e Mo via foliar.

Para as características número de vagens por planta e número de sementes por planta, foram observadas diferenças significativas pelo teste $\mathrm{F}(\mathrm{P} \leq 0,05)$, sendo que o tratamento com inoculante apresentou-se superior, onde se obteve incrementos de $8,4 \%$ e $9,5 \%$ para número de vagens por planta e número de sementes por planta, respectivamente (Tabela 2).

Na Tabela 2 estão apresentados os resultados para massa de 100 sementes e produtividade da cultura da soja com e sem a aplicação de inoculante. Os dados contidos nessa Tabela demonstram que o tratamento com inoculante comercial não promoveu aumentos significativos nas características descritas. Resultados semelhantes foram obtidos por Gris et al. (2005) que não obtiveram resultados significativos na produtividade da soja com a inoculação das sementes com Bradyrhizobium japonicum em um Latossolo Vermelho eutroférrico em condições de plantio direto no município de Palotina (PR). Campos et al. (2001) também não constataram resposta à reinoculação em soja manejada em sistema plantio direto.

Em relação a não existência de diferenças significativas entre sementes tratadas e as não tratadas com inoculante comercial, é provável que o solo já disponha de populações estabelecidas de bactérias que proporcionaram boa nodulação e fixação biológica do $\mathrm{N}_{2}$, considerando-se que, nessa área, vem sendo cultivada a soja nos últimos oito anos em sistema de cultivo mínimo e onde foram realizadas inoculações do rizóbio.

Mesmo não ocorrendo efeitos significativos com a aplicação do inoculante comercial, a produtividade de sementes da soja, aumentou de $3.242,9 \mathrm{~kg} \mathrm{ha}^{-1}$, no tratamento sem inoculante, para $3.345,5 \mathrm{~kg} \mathrm{ha}^{-1}$, com a inoculação das sementes, aumentando, portanto, em 102,6 $\mathrm{kg} \mathrm{ha}^{-1}$ a produção de sementes com esta inoculação, o equivalente a $3,2 \%$ ou 1,7 sc ha $^{-1}$, próximo do obtido pela Embrapa Soja (2006), que relata ganhos médios de 4,5\% em função da reinoculação em áreas já cultivadas com soja. Assim, mesmo não apresentando resultados significativos, a inoculação das sementes da cultura da soja pode representar ganhos econômicos, uma vez que o custo deste produto é baixo, em torno de $\mathrm{R} \$ 2,50 \mathrm{ha}^{-1}$ (ou cerca de $11 \%$ do valor de uma saca de soja comercial) e, considerando-se que a grande maioria dos produtores realiza o tratamento das sementes com fungicidas ou inseticidas, e esta operação pode ser realizada em seqüência.

Para a característica número de sementes por vagem, tanto quando submetida à aplicação do inoculante quanto as 
diferentes doses de CoMo, não foram observadas diferenças significativas pelo Teste $\mathrm{F}$ (Tabela 2). A não variabilidade para esta característica parece estar mais ligada ao genótipo utilizado do que aos fatores nutricionais.

TABELA 2. Valores de F, coeficiente de variação (C.V.), diferença mínima significativa (DMS) e teste de Tukey para altura de planta (AP), altura de inserção de $1^{\text {a }}$ vagem (AIV), número de vagens por planta (NVP), número de sementes por planta (NSP), número de sementes por vagem (NSV), massa de 100 sementes (M100S), produtividade (P), germinação (G), índice de velocidade de germinação (IVG), primeira contagem (PC) e teste de frio (TF) da cultura da soja, cv. MSOY 8411 submetida a diferentes doses de cobalto e molibdênio e inoculação com Bradyrhizobium japonicum, no município de Santa Carmem, MT (2005/2006).

\begin{tabular}{|c|c|c|c|c|c|c|c|c|c|c|c|}
\hline & $\begin{array}{l}\mathrm{AP} \\
(\mathrm{cm})\end{array}$ & $\begin{array}{l}\text { AIV } \\
(\mathrm{cm})\end{array}$ & $\begin{array}{l}\text { NVP } \\
\left(\mathrm{n}^{\circ}\right)\end{array}$ & $\begin{array}{l}\text { NSP } \\
\left(\mathrm{n}^{\circ}\right)\end{array}$ & $\begin{array}{l}\mathrm{NSV} \\
\left(\mathrm{n}^{\circ}\right)\end{array}$ & $\begin{array}{l}\text { M100S } \\
(\mathrm{g})^{1}\end{array}$ & $\begin{array}{c}\mathrm{P} \\
\left(\mathrm{kg} \mathrm{ha}^{-1}\right)^{1}\end{array}$ & $\begin{array}{c}\mathrm{G} \\
(\%)\end{array}$ & $\begin{array}{l}\text { IVG } \\
(\%)\end{array}$ & $\begin{array}{l}\mathrm{PC} \\
(\%)\end{array}$ & $\begin{array}{l}\mathrm{TF} \\
(\%)\end{array}$ \\
\hline \multicolumn{12}{|l|}{ Inoculante } \\
\hline Com & $59,8 \mathrm{a}$ & $14,9 \mathrm{a}$ & $64,8 \mathrm{a}$ & $132,3 \mathrm{a}$ & $2,0 \mathrm{a}$ & $15,6 \mathrm{a}$ & $3345,5 \mathrm{a}$ & $84,2 \mathrm{a}$ & $16,3 \mathrm{a}$ & $76,8 \mathrm{a}$ & $53,3 \mathrm{a}$ \\
\hline Sem & $60,6 \mathrm{a}$ & $14,9 \mathrm{a}$ & $59,7 \mathrm{~b}$ & $120,9 \mathrm{~b}$ & $2,0 \mathrm{a}$ & $15,4 \mathrm{a}$ & $3242,9 \mathrm{a}$ & $85,2 \mathrm{a}$ & $16,4 \mathrm{a}$ & $76,4 \mathrm{a}$ & $51,5 \mathrm{a}$ \\
\hline $\mathrm{F}$ & $0,42 \mathrm{~ns}$ & $0,0009 \mathrm{~ns}$ & $4,37 *$ & $5,47 *$ & $0,42 \mathrm{~ns}$ & $1,18 \mathrm{~ns}$ & $3,21 \mathrm{~ns}$ & $0,38 \mathrm{~ns}$ & $\begin{array}{c}0,10 \\
\mathrm{~ns}\end{array}$ & $0,04 \mathrm{~ns}$ & $0,59 \mathrm{~ns}$ \\
\hline DMS (5\%) & 2,35 & 0,85 & 4,89 & 9,96 & 0,05 & 0,36 & 116,5 & 3,28 & 0,64 & 3,53 & 4,61 \\
\hline \multicolumn{12}{|l|}{$\begin{array}{l}\text { Doses de CoMo } \\
\left(\mathrm{L} \mathrm{h} \mathrm{h}^{-1}\right)\end{array}$} \\
\hline 0,00 & 62,4 & 15,7 & 59,3 & 123,7 & 2,1 & 15,0 & 3183,7 & 88,3 & 17,1 & 81,3 & 56,3 \\
\hline 0,25 & 61,2 & 14,6 & 64,5 & 130,2 & 2,0 & 15,8 & 3397,6 & 83,3 & 15,9 & 73,3 & 59,5 \\
\hline 0,50 & 59,8 & 14,7 & 58,7 & 120,0 & 2,0 & 15,8 & 3227,0 & 82,5 & 15,7 & 71,3 & 53,0 \\
\hline 1,00 & 58,0 & 13,9 & 66,6 & 131,5 & 2,0 & 15,3 & 3176,5 & 86,0 & 16,7 & 79,5 & 56,3 \\
\hline 1,50 & 60,4 & 15,3 & 66,0 & 133,1 & 2,0 & 15,9 & 3481,5 & 83,3 & 16,0 & 74,0 & 49,5 \\
\hline 2,00 & 59,3 & 15,4 & 58,3 & 121,2 & 2,1 & 15,3 & 3299,1 & 84,8 & 16,6 & 79,8 & 39,8 \\
\hline $\mathrm{F}$ & $1,19 \mathrm{~ns}$ & $1,57 \mathrm{~ns}$ & $1,72 \mathrm{~ns}$ & $0,89 \mathrm{~ns}$ & $1,62 \mathrm{~ns}$ & $2,96 *$ & $3,11 *$ & $1,20 \mathrm{~ns}$ & $2,11 \mathrm{~ns}$ & $3,59 * *$ & $6,44 * *$ \\
\hline $\begin{array}{c}\text { Inoc x CoMo } \\
\text { F }\end{array}$ & $0,60 \mathrm{~ns}$ & $0,39 \mathrm{~ns}$ & $0,91 \mathrm{~ns}$ & $1,20 \mathrm{~ns}$ & $1,02 \mathrm{~ns}$ & $0,68 \mathrm{~ns}$ & $0,77 \mathrm{~ns}$ & $4,10 * *$ & $4,80 * *$ & $4,81 * *$ & $4,61 * *$ \\
\hline C. V. $\%$ & 6,64 & 9,77 & 13,37 & 13,38 & 4,59 & 3,96 & 6,01 & 6,60 & 6,68 & 7,87 & 15,01 \\
\hline
\end{tabular}

${ }^{1}$ Com base em $13 \%$ de umidade nas sementes.

Valores seguidos pelas mesmas letras minúsculas nas colunas não diferem entre si a 5\% de probabilidade pelo teste de Tukey.

${ }^{\text {ns }}$ Valores não diferem segundo o Teste F.

* Médias significativas a 5\% de probabilidade segundo o Teste F.

** Médias significativas a $1 \%$ de probabilidade segundo o Teste $\mathrm{F}$.

Como pode ser observado na Tabela 2, não houve interação significativa entre inoculante X CoMo para altura de planta, altura de inserção de $1^{\circ}$ vagem, número de vagens por planta, número de sementes por planta, número de sementes por vagem, massa de 100 sementes e produtividade. De acordo com o dados contidos nessa tabela, verifica-se que as doses de CoMo influenciaram significativamente as características massa de 100 sementes e produtividade.
A representação gráfica do efeito das doses de CoMo em aplicação via foliar sobre a variável massa de 100 sementes pode ser observada na Figura 1, onde o maior valor obtido se deu na dose de $1,5 \mathrm{~L} \mathrm{ha}^{-1}$ p.c.com a média de $15,90 \mathrm{~g}$, e a menor média ocorreu na testemunha $\left(0,0 \mathrm{~L} \mathrm{ha}^{-1}\right.$ p.c. $)$ com massa de 15,01 g. Observa-se que a dose de 1,5 L ha-1 p.c., provocou um aumento de 5,9\% na massa de 100 sementes, em comparação à testemunha. (0,0 L ha-1 p.c.). 


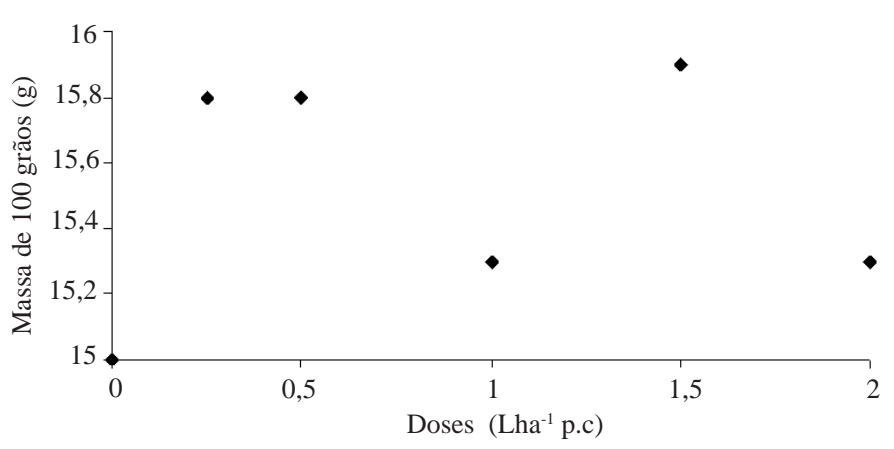

FIGURA 1. Massa de 100 grãos da cultura da soja, cv.MSOY 8411, submetida a diferentes doses de CoMo em aplicação via foliar, no município de Santa Carmem, MT (2005/2006).

Na Figura 2 encontra-se a representação gráfica do comportamento da produtividade da cultura da soja em função das doses de CoMo aplicadas via foliar. A maior produtividade ocorreu na dose de $1,5 \mathrm{~L} \mathrm{ha}^{-1}$ p.c. de CoMo, sendo que essa foi de $3.481,5 \mathrm{~kg} \mathrm{ha}^{-1}$. Já os menores valores foram observados nas doses de 1,0 e 0,0 L ha-1 p.c. de CoMo, com médias de $3.176,5$ e $3.183,7 \mathrm{~kg} \mathrm{ha}^{-1}$. Verifica-se que a dose de $1,5 \mathrm{~L} \mathrm{ha}^{-1}$ p.c., provocou um aumento de 9,4\% na produtividade de sementes da cultura da soja, em comparação a testemunha (0,0 L ha-1 p.c.).

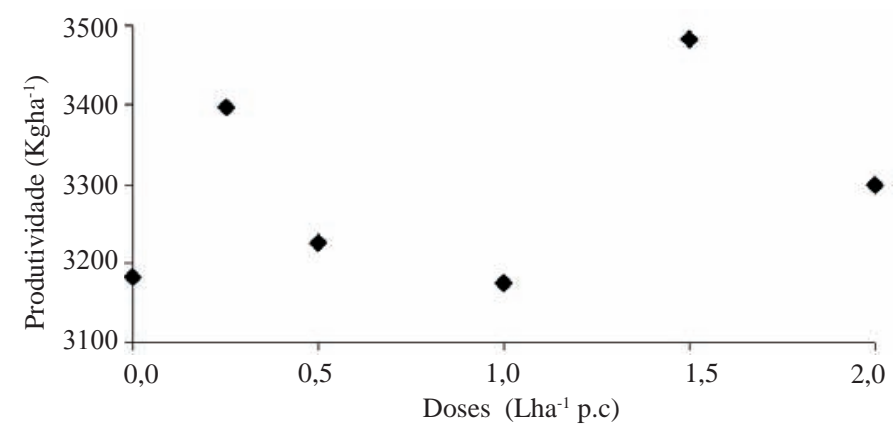

FIGURA 2. Produtividade de soja, cv. MSOY 8411, obtida sob condições de diferentes doses de CoMo em aplicação via foliar, no município de Santa Carmem, MT (2005/2006).

Esses resultados assemelham-se com os obtidos por Meschede et al. (2004), que obtiveram um incremento de $20 \%$ da produtividade da soja em relação ao controle, através da adubação foliar com Comol (produto comercial contendo $1 \%$ de $\mathrm{Co}+10 \%$ de $\mathrm{Mo}$ ), concordam também com Santos (1991) que relata aumentos de até 32,7\% pela utilização de doses crescentes $\left(0,100,200,300\right.$ e $\left.400 \mathrm{~g} \mathrm{ha}^{-1}\right)$ de um produto comercial contendo $10 \%$ de Mo e $1 \%$ de Co.

Como pode ser observado nas Figuras 1 e 2, a aplicação de CoMo, por via foliar, ocasionou aumento na massa de 100 sementes e na produtividade dessa cultura. Os valores máximos foram obtidos para o tratamento de $1,5 \mathrm{~L} \mathrm{ha}^{-1}$ do p.c. Esses resultados podem ser explicados pela participação do Mo como componente do complexo enzimático denominado nitrogenase que catalisa a fixação do $\mathrm{N}_{2}$ no bacterióide, promovendo maior atividade na fixação do $\mathrm{N}_{2}$, bem como de atuar como constituinte da enzima nitrato redutase, a qual reduz íons nitrato a nitrito, possibilitando sua incorporação em compostos orgânicos nas raízes e na parte aérea (Marschner, 1986). Além disso, o Co é um elemento essencial para o processo de FBN. Ele é componente da vitamina $\mathrm{B}_{12}$, importante na formação da coenzima cobamida que é indispensável ao processo de FBN por ser precursora da leghemoglobina (Kliewer e Evans, 1963).

Na Tabela 2 encontram-se os resultados das análises estatísticas para Germinação (\%), IVG (Índice de Velocidade de Germinação) (\%), Primeira contagem (\%) e Teste Frio (\%) das sementes da cultura da soja produzidas sob diferentes doses de Cobalto e Molibdênio (CoMo), com e sem a inoculação com Bradyrhizobium japonicum.

Com os resultados obtidos para germinação de sementes de soja, apresentados na Tabela 2, podemos verificar que esses se encontram acima dos padrões de germinação mínima exigida, que é de $75 \%$ de acordo com os padrões descritos pela Embrapa Soja (2006).

Como se observa na Tabela 2, todas as características testadas, referentes a qualidade fisiológica de sementes, apresentaraminteração significativa entre os fatores inoculante e doses de CoMo $(\mathrm{P}<0,01)$. Na Tabela 3, são apresentados os desdobramentos referentes a estas interações.

Esses resultados diferem dos obtidos por Harris et al. (1965), que em trabalhos visando verificar o efeito de molibdênio sobre a qualidade das sementes produzidas em soja, não verificou a ação deste nutriente sobre a qualidade das sementes nos diversos locais em que os experimentos foram realizados.

Na Figura 3 encontra-se a representação gráfica da germinação no desdobramento da interação entre os fatores inoculante e doses de CoMo. Podemos observar o comportamento do fator doses de CoMo dentro de inoculação. De acordo com os dados, verifica-se que a maior média de germinação foi obtida, quando não realizou-se a inoculação, se deu na dose de $0,0 \mathrm{~L} \mathrm{ha}^{-1}$, com média 92,50\%, sendo que a germinação diminuiu conforme as dose de CoMo foram aumentadas e assim a dose de 2,0 L ha-1 apresentou a menor 
germinação (80,00\%). Já quando realizada a inoculação, a maior germinação foi obtida na dose $2,0 \mathrm{~L} \mathrm{ha}^{-1}$ com média de $89,50 \%$, e as menores médias foram obtidas nas doses de 0,25 e 0,5 L ha-1 de CoMo, com 79,00\% de germinação.

TABELA 3. Desdobramento das interações significativas entre inoculante e doses de CoMo para germinação (\%), IVG (\%), primeira contagem (\%) e teste de frio (\%) das sementes de soja da cv. MSOY 8411, produzidas sob condições de diferentes doses de CoMo, com e sem a inoculação com Bradyrhizobium japonicum, no município de Santa Carmem, MT (2005/2006).

\begin{tabular}{|c|c|c|c|c|c|c|}
\hline \multirow{3}{*}{ Inoculante } & \multicolumn{6}{|c|}{ Germinação (\%) } \\
\hline & \multicolumn{6}{|c|}{ Doses de CoMo $\left(\mathrm{L} \mathrm{ha}^{-1}\right)$} \\
\hline & 0,0 & 0,25 & 0,5 & 1,0 & 1,5 & 2,0 \\
\hline Com & $84,0 \mathrm{~b}$ & $79,0 \mathrm{~b}$ & $79,0 \mathrm{a}$ & 89,0 a & $84,5 \mathrm{a}$ & $89,5 \mathrm{a}$ \\
\hline \multirow[t]{3}{*}{ Sem } & $92,5 \mathrm{a}$ & $87,5 \mathrm{a}$ & 86,0 a & $83,0 \mathrm{a}$ & 82,0 a & $80,0 \mathrm{~b}$ \\
\hline & \multicolumn{6}{|c|}{$\operatorname{IVG}(\%)$} \\
\hline & \multicolumn{6}{|c|}{ Doses de CoMo $\left(\mathrm{L} \mathrm{ha}^{-1}\right)-$} \\
\hline Com & $16,2 \mathrm{~b}$ & $15,0 \mathrm{~b}$ & $15,1 \mathrm{a}$ & $17,5 \mathrm{a}$ & $16,3 \mathrm{a}$ & $17,5 \mathrm{a}$ \\
\hline \multirow[t]{3}{*}{ Sem } & $18,0 \mathrm{a}$ & $16,8 \mathrm{a}$ & $16,3 \mathrm{a}$ & $15,9 \mathrm{~b}$ & $15,6 \mathrm{a}$ & $15,6 \mathrm{~b}$ \\
\hline & \multicolumn{6}{|c|}{ Primeira Contagem $(\%)$} \\
\hline & \multicolumn{6}{|c|}{$\longrightarrow$ Doses de CoMo $\left(\mathrm{L} \mathrm{ha}^{-1}\right) \longrightarrow$} \\
\hline Com & $76,5 \mathrm{~b}$ & $68,5 \mathrm{~b}$ & 69,5 a & 85,0 a & 76,5 a & $84,5 \mathrm{a}$ \\
\hline \multirow[t]{3}{*}{ Sem } & 86,0 a & $78,0 \mathrm{a}$ & $74,0 \mathrm{a}$ & $74,0 \mathrm{~b}$ & $71,5 \mathrm{a}$ & $75,0 \mathrm{~b}$ \\
\hline & \multicolumn{6}{|c|}{ Teste Frio $(\%)$} \\
\hline & \multicolumn{6}{|c|}{ Doses de CoMo $\left(\mathrm{L} \mathrm{ha}^{-1}\right)-$} \\
\hline Com & $53,5 \mathrm{a}$ & $53,0 \mathrm{~b}$ & $55,0 \mathrm{a}$ & 56,0 a & $51,0 \mathrm{a}$ & $51,0 \mathrm{a}$ \\
\hline Sem & $59,0 \mathrm{a}$ & $66,0 \mathrm{a}$ & $51,0 \mathrm{a}$ & $56,5 \mathrm{a}$ & $48,0 \mathrm{a}$ & $28,5 \mathrm{~b}$ \\
\hline
\end{tabular}

Valores seguidos pela mesma letras nas colunas, dentro de cada parâmetro, não diferem entre si a 5\% de probabilidade pelo teste de Tukey

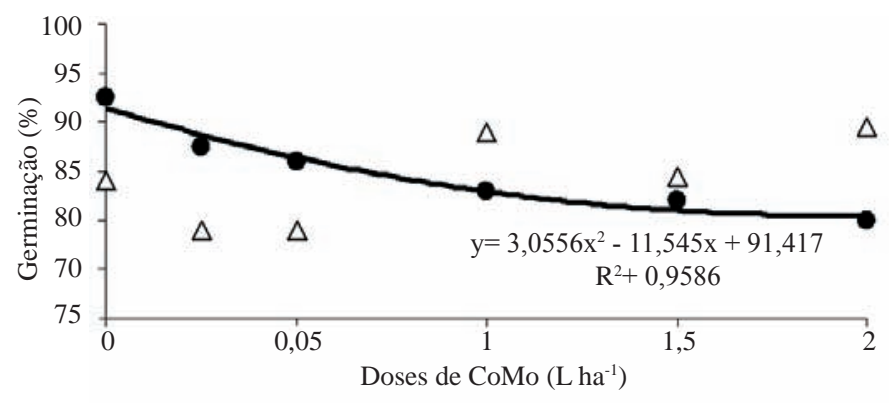

$\Delta$ Com Inoc. $\quad$ Sem Inoc.

FIGURA 3. Germinação de sementes de soja, cv. MSOY 8411, em função da interação significativa entre inoculante e doses de CoMo da cultura da soja submetida a diferentes doses de CoMo, em aplicação foliar, com e sem inoculação com Bradyrhizobium japonicum no município de Santa Carmem, MT (2005/2006).
Estes resultados diferem dos obtidos por Ambrosano et al. (1999), que avaliando o efeito da adubação nitrogenada e micronutrientes na qualidade de sementes do feijoeiro cultivar IAC - Carioca, observaram que os tratamentos não influenciaram a percentagem de germinação e de plântulas anormais, tanto nos ensaios de aplicação de nitrogênio como nos de micronutrientes, não tendo estes alterado a qualidade das sementes determinada pelo teste de germinação.

A representação gráfica do desdobramento da interação entre inoculante e doses de CoMo para IVG pode ser observada na Figura 4. Observa-se que quando não foi realizada a inoculação a maior e menor média para IVG foram obtidas nas doses de 0,0 e 1,5 $\mathrm{L} \mathrm{ha}^{-1}$ respectivamente. Nesta mesma Figura, pode-se verificar o comportamento de doses de CoMo quando se utilizou o inoculante. Neste caso, a maior média foi encontrada na dose de 2,0 $\mathrm{L} \mathrm{ha}^{-1} \mathrm{com}$ $17,53 \%$, e a menor média obtida $(15,01 \%)$ ocorreu na dose de $0,25 \mathrm{~L} \mathrm{ha}^{-1}$. 


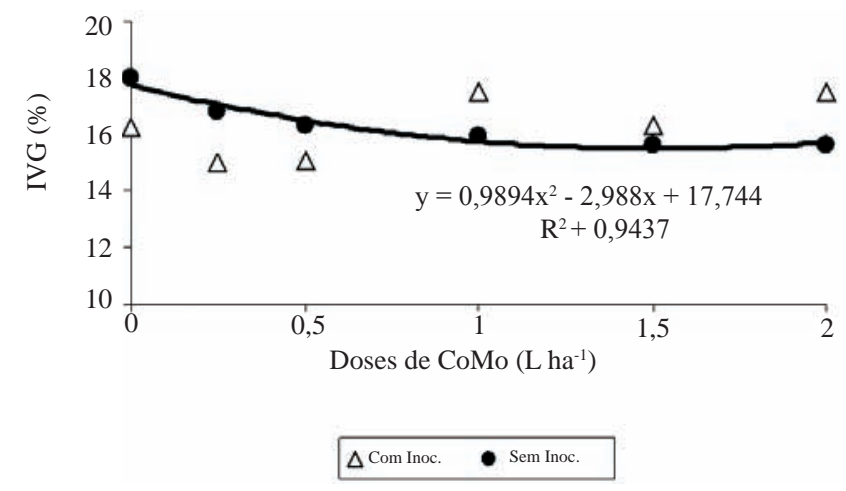

FIGURA 4. IVG de sementes de soja da cv. MSOY 8411, em função da interação significativa entre inoculante e doses de CoMo da cultura da soja, submetida a diferentes doses de CoMo, em aplicação foliar, com e sem inoculação com Bradyrhizobium japonicum no município de Santa Carmem, MT (2005/2006).

Na Figura 5 pode ser observado o desdobramento da interação entre inoculante e doses de CoMo para o teste de Primeira Contagem. Com inoculação, a maior média foi obtida na dose $1,0 \mathrm{~L} \mathrm{ha}^{-1}(85,00 \%)$ e para sem, na dose de 0,0 $\mathrm{L} \mathrm{ha}^{-1}$ foi verificada a maior média $(86,00 \%)$.

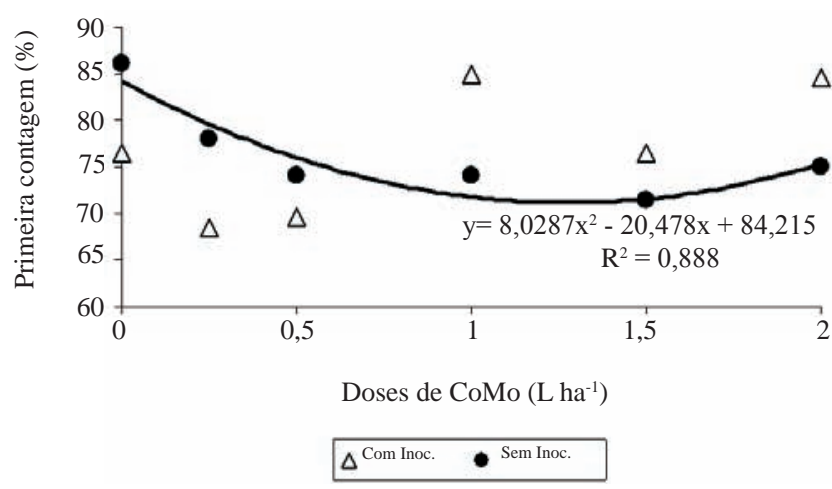

FIGURA 5. Resultados do teste de primeira contagem de sementes de soja da cv. MSOY 8411, em função da interação significativa entre inoculante e doses de CoMo da cultura da soja, submetida a diferentes doses de CoMo em aplicação foliar, com e sem inoculação com Bradyrhizobium japonicum no município de Santa Carmem, MT (2005/2006).

$\mathrm{Na}$ Figura 6 esta apresentado graficamente o desdobramento da interação entre doses de CoMo e inoculação para o Teste Frio. No que se refere ao tratamento com inoculante, a maior média para o teste frio foi obtida com a dose de 1,0 L ha-1 com 56,00\%. Sem a inoculação, a maior média foi observada na dose de $0,25 \mathrm{~L} \mathrm{ha}^{-1}$.

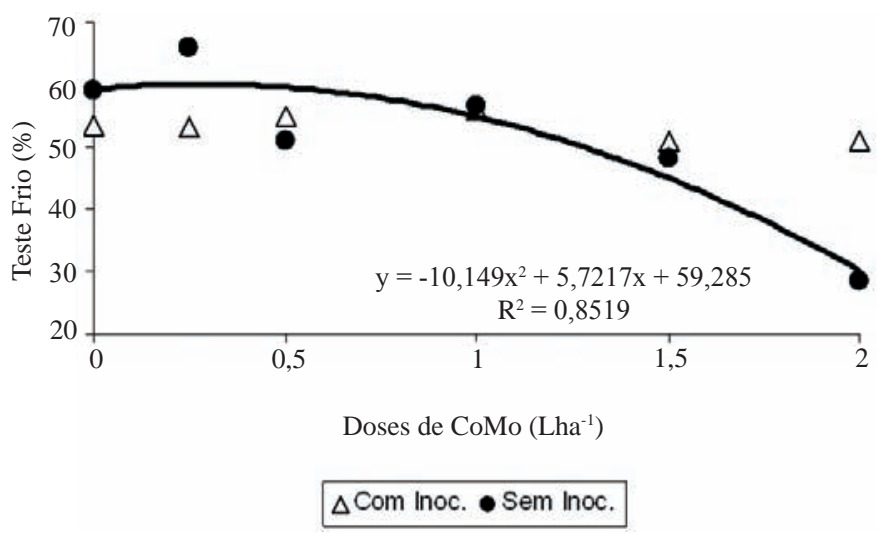

FIGURA 6. Resultados do teste de frio realizado de sementes de soja da cv. MSOY 8411, em função da interação significativa entre inoculante e doses de CoMo da cultura da soja, submetida a diferentes doses de CoMo, em aplicação foliar, com e sem inoculação com Bradyrhizobium japonicum, no município de Santa Carmem, MT (2005/2006).

De acordo com os dados apresentados nas Figuras 3, 4, 5 e 6, onde estão representados graficamente a germinação, IVG, Primeira Contagem e Teste Frio, das sementes da cultura da soja em função da interação entre inoculante e doses de CoMo, podemos observar que em todas estas variáveis, quando não é realizada a inoculação, as médias tenderam a ser diminuídas com o aumento da doses de CoMo. Diferentemente, o comportamento das diferentes doses de CoMo, quando foi realizada a inoculação, se apresentaram bastante variáveis, não tendo uma tendência de aumento ou diminuição de acordo com as doses.

\section{CONCLUSÕES}

Coma base nos resultados obtidos e nas condições em que foi desenvolvido o presente trabalho pode-se concluir que:

a) a inoculação das sementes com Bradyrhizobium 
japonicum aumenta o número de vagens por planta e o número de sementes por planta;

b) as doses de CoMo influenciam significativamente a massa de 100 sementes e a produtividade de sementes da cultura, sendo a dose de $1,5 \mathrm{~L} \mathrm{ha}^{-1}$ p.c. a que apresenta os maiores valores;

c) ocorrem interações significativas entre os fatores inoculante e doses de CoMo para as características germinação, IVG, primeira contagem e teste frio. A aplicação de $2 \mathrm{~L} \mathrm{ha}^{-1}$ de CoMo em sementes inoculadas promove melhoria na qualidade fisiológica das sementes. Na ausência de inoculação, o aumento da doses de CoMo ocasiona diminuição na qualidade fisiológica das sementes.

\section{REFERÊNCIAS}

AMBOSANO, E. J.; AMBROSANO, G. M. B.; WUTKE, E. B.; BULISANI, E. A.; MARTINS, A. L. M.; SILVEIRA, L. C. P. Efeitos da adubação nitrogenada e com micronutrientes na qualidade de sementes do feijoeiro cultivar IAC - Carioca. Bragantina, v.58, n. 2, 1999.

BARROS, A.S.R. Teste de frio. In: KRZYZANOWSKI, F.C. et al. (Ed.). Vigor de sementes: conceitos e testes. Londrina: Abrates, 1999. cap. 5, p. 1-15.

BRASIL, Ministério da Agricultura e Reforma Agrária. Regras para análises de sementes. Brasília, DF: SNAD; CLAV, 1992.

CAMPOS, B.C, HUNGRIA, M.; TEDESCO, V.. Eficiência da fixação biológica de $\mathrm{N}_{2}$ por estirpes de Bradyrhizobium na soja em plantio direto. In: Revista Brasileira de Ciência do Solo, v. 25, p. 583-592. Viçosa, 2001.

CARVALHO, M. A. C. de; LAZARINI, E.; SÁ, M. E. de; OLIVEIRA, A. L. de. Variações na metodologia do teste de frio para avaliação do vigor de sementes de soja. Revista Brasileira de Sementes, v.22, n.1, p. 74-80, 2000.

CARVALHO, N.M.; NAKAGAWA, J. Sementes: ciência, tecnologia e produção. 4. ed. Jaboticabal: Funep, 2000. 588p.

TECNOLOGIAS de produção de soja - região central do Brasil 2007. Londrina: Embrapa Soja: Embrapa Cerrados: Embrapa Agropecuária Oeste, 2006. 225p. (Embrapa Soja. Sistema de Produção, 11).

GOMES, F. P. A estatística moderna na pesquisa agropecuária. Piracicaba: POTAFÓS, 1984, 160 p.

GRIS, E. P.; CASTRO, A. M. C. e; OLIVEIRA, F. F. de. Produtividade da soja em resposta à aplicação de molibdênio e inoculação com Bradyrhizobium japonicum. In: Revista Brasileira de Ciência do Solo, v. 29 p. 151-155. Viçosa, 2005.

HARRIS, H. C.; PARKER, M. B.; JHONSON, B. J. Influence of molybdenum content of soybean seed and other factors associated with source on progeny response to applud molybdenum. Agronomy Journal, v. 57, n. 2, p. 397 - 399, 1965.

KLIEWER, M. ; EVANS, H. J. Cobamide coenzyme contents of soybean nodules and nitrogen fixing bacteria in relation to physiological conditions. Plant Physiology, v. 38, p $99-$ 104, 1963.

MARCOS-FILHO, J.; CÍCERO, S.M.; SILVA, W.R. Avaliação da qualidade das sementes. Piracicaba: FEALQ, 1987. 230p.

MARSCHNER, H. Mineral nutrition of higher plants. London, Academic Press, 1986. 674 p.

MENGEL, K.; KIRKBY, E.A. Principles of plant nutrition. 5 ed. Dordrecht: Kluwer Academic Publishers, 2001. 849p.

MESCHEDE D. K.; BRACCINI, A. de L. e; BRACCINI, M. do C. L.; SCAPIM, C. A.; SCHUAB, S. R. P. Rendimento, teor de proteínas nas sementes e características agronômicas das plantas de soja em resposta à adubação foliar e ao tratamento de sementes com molibdênio e cobalto. Acta Scientiarum. Agronomy, v. 26, no. 2, p. 139-145, 2004.

NOVAIS, R. F. de. Soja. In: RIBEIRO, A. C.; GUIMARÃES, P. T.; ALVAREZ V., V. H. Recomendações para o uso de corretivos e fertilizantes em Minas Gerais - $5^{\circ}$ Aproximação. Viçosa: 1999, 359 p.

ROCHA, V. S.; OLIVEIRA, A. B.; SEDIYAMA, T.; GOMES J. L. L.; SEDIYAMA C. S.; PEREIRA, M. G. A qualidade da semente de soja. Viçosa, UFV, 1984. 76 p.

SÁ, M.E. Importância da adubação na qualidade de sementes. In: SÁ, M.E. e BUZZETI, S. (Coord.). Importância da adubação na qualidade dos produtos agrícolas. São Paulo: Ícone, 1994. p. 65-98.

SANTOS, O. S. Molibdênio. In: FERREIRA M.E.; CRUZ, M.C.P. (Ed.). Micronutrientes na agricultura. Piracicaba: Potafos/CNPq, 1991. p.191-217.

SFREDO, G.J.; BORKERT, C.M.; LANTAMANN, A.F.; MEYER, M.C.; MANDARINO, J.M.G.; OLIVEIRA, M.C.N. Molibdênio e cobalto na cultura da soja. Londrina: Embrapa- CNPSo, 1997. 18p. (Embrapa- CNPSo. Circular Técnica, 16).

VARGAS, M. A. T.; SUHET, A. R.; MENDES, I. de C.; PERES, J. R. Fixação biológica de nitrogênio em solos de 
cerrados. Brasília: EMBRAPA - CPAC: EMBRAPA - SPI, 1994. 83 p.
VIEIRA, R. D.; CARVALHO, N. M. Teste de vigor em sementes. Jaboticabal: FUNEP, 1994. p. 164. 